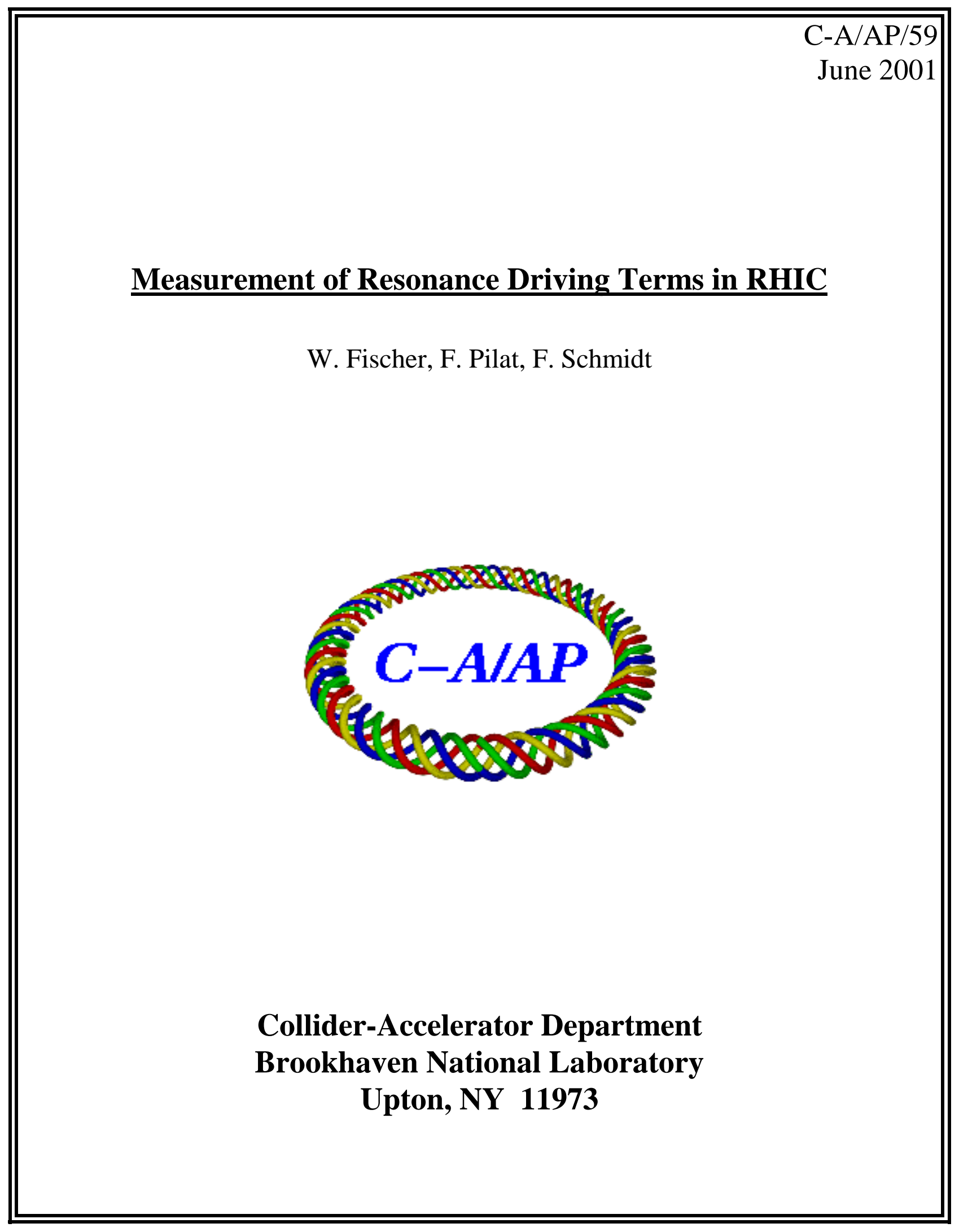




\title{
Measurement of Resonance Driving Terms in RHIC
}

\author{
W. Fischer, F. Pilat, BNL, and F. Schmidt, CERN
}

June 28, 2001

\begin{abstract}
The RHIC interaction region (IR) correction system, consisting of multi-layer correction packages located next to the IR triplets and independently powered, has been commissioned during the year 2000 run. A frequency analysis technique has been tested as a beam-based method to set the IR correctors. It minimizes the resonance driving terms, caused by field errors, order by order. Beam measurements of the run 2000 are presented and discussed.
\end{abstract}

\section{Introduction}

In the recent past, new techniques were developed that allow a more precise determination of the tunes $[1,2]$. These methods can also be used to other find spectral lines in descending order of magnitude from turn-by-turn beam position data. It seems therefore appropriate to review the connection between experimental data and theoretical models as first attempted in 1988 [3].

As demonstrated in Ref. [4] one can link spectral lines to the components of the distortion function or interpret them as resonances. It is also shown in this paper that all excited resonances can be determined in a order-by-order process. More recently, this method has been successfully applied in controlled experiments at the SPS [5]. It seems therefore an useful analysis tool in the commissioning phase of an accelerator. The tool assists in locating multipole errors and setting correctors to counteract. Last year, a first attempt was made to apply this technique at RHIC.

\section{Some Theory}

The theory has been developed in depth in Ref. [4]. A short outline follows in this section. The complex Fourier spectrum of the normalized coordinates $\left(\hat{x}, \hat{p}_{x}\right)$ can be written as

$$
\begin{aligned}
\hat{x}(N)-i \hat{p}_{x}(N)= & \sum_{j=1}^{\infty} a_{j} e^{i\left[2 \pi\left(m_{j} \nu_{x}+n_{j} \nu_{y}\right) N+\psi_{j}\right]} \\
& m_{j}, n_{j} \in \mathbf{Z},
\end{aligned}
$$

where $N$ is the turn number and $\nu_{x}$ and $\nu_{y}$ are the tunes. The connection between oneturn maps and Normal Form can be conveniently described using the Map - Normal Form Diagram (for details see [6]):

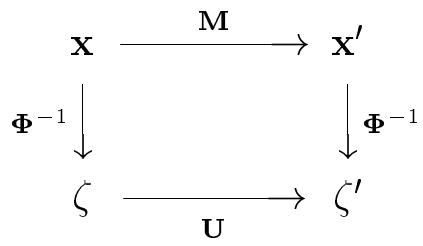


Table 1: Generating function (theory) compared to spectra lines (experiment)

\begin{tabular}{|c|c|c|}
\hline & $\begin{array}{c}\text { Generating } \\
\text { Function } \\
\end{array}$ & $\begin{array}{c}\text { Spectral } \\
\text { Line }\end{array}$ \\
\hline Amplitude & $\left|f_{j k l m}\right|$ & $\begin{array}{l}\left|H S L_{j k l m}\right|=2 \cdot j \cdot\left(2 I_{x}\right)^{\frac{j+k-1}{2}}\left(2 I_{y}\right)^{\frac{l+m}{2}}\left|f_{j k l m}\right| \\
\left|V S L_{j k l m}\right|=2 \cdot l \cdot\left(2 I_{x}\right)^{\frac{j+k}{2}}\left(2 I_{y}\right)^{\frac{l+m-1}{2}}\left|f_{j k l m}\right|\end{array}$ \\
\hline Phase & $\phi_{j k l m}$ & $\begin{array}{l}P H S L_{j k l m}=\phi_{j k l m}+(1-j+k) \psi_{x_{0}}-(l-m) \psi_{y_{0}}-\frac{\pi}{2} \\
P V S L_{j k l m}=\phi_{j k l m}-(j-k) \psi_{x_{0}}+(1-l+m) \psi_{y_{0}}-\frac{\pi}{2}\end{array}$ \\
\hline
\end{tabular}

The generating function $F$ and the Hamiltonian $H$ are given by

$$
\boldsymbol{\Phi}=e^{: F(J, \phi):} \quad, \quad \mathbf{U}=e^{: H(J):} .
$$

The Normal Form coordinates can then be written as

$$
\zeta=e^{-: F_{r}:} \mathbf{h}, \quad h_{z}^{ \pm}=\hat{z} \pm i \hat{p}_{z},
$$

with the generating function in resonance basis as

$$
F_{r}=\sum_{j k l m} f_{j k l m} \zeta_{x}^{+j} \zeta_{x}^{-k} \zeta_{y}^{+l} \zeta_{y}^{-m}
$$

or

$$
\begin{aligned}
& F_{r}=\sum_{j k l m} f_{j k l m}\left(2 I_{x}\right)^{\frac{j+k}{2}}\left(2 I_{y}\right)^{\frac{l+m}{2}} \times \\
& e^{-i\left[(j-k)\left(\psi_{x}+\psi_{x_{0}}\right)+(l-m)\left(\psi_{y}+\psi_{y_{0}}\right)\right]} .
\end{aligned}
$$

The generating function in action leads to

$$
\mathbf{h}=e^{: F_{r}:} \zeta=\zeta+\left[F_{r}, \zeta\right]+\frac{1}{2}\left[F_{r},\left[F_{r}, \zeta\right]\right]+\cdots
$$

and the evolution of linearly normalized coordinates can be expressed as

$$
\begin{aligned}
& h_{x}^{-}(N)=\sqrt{2 I_{x}} e^{i\left(2 \pi \nu_{x} N+\psi_{x_{0}}\right)} \\
& -2 i \sum_{j k l m} j f_{j k l m}\left(2 I_{x}\right)^{\frac{j+k-1}{2}}\left(2 I_{y}\right)^{\frac{l+m}{2}} \times \\
& e^{i\left[(1-j+k)\left(2 \pi \nu_{x} N+\psi_{x_{0}}\right)+(m-l)\left(2 \pi \nu_{y} N+\psi_{y_{0}}\right)\right]} .
\end{aligned}
$$

As a consequence, the terms of the generating function and the spectral lines are related as shown in Tab. 1. 

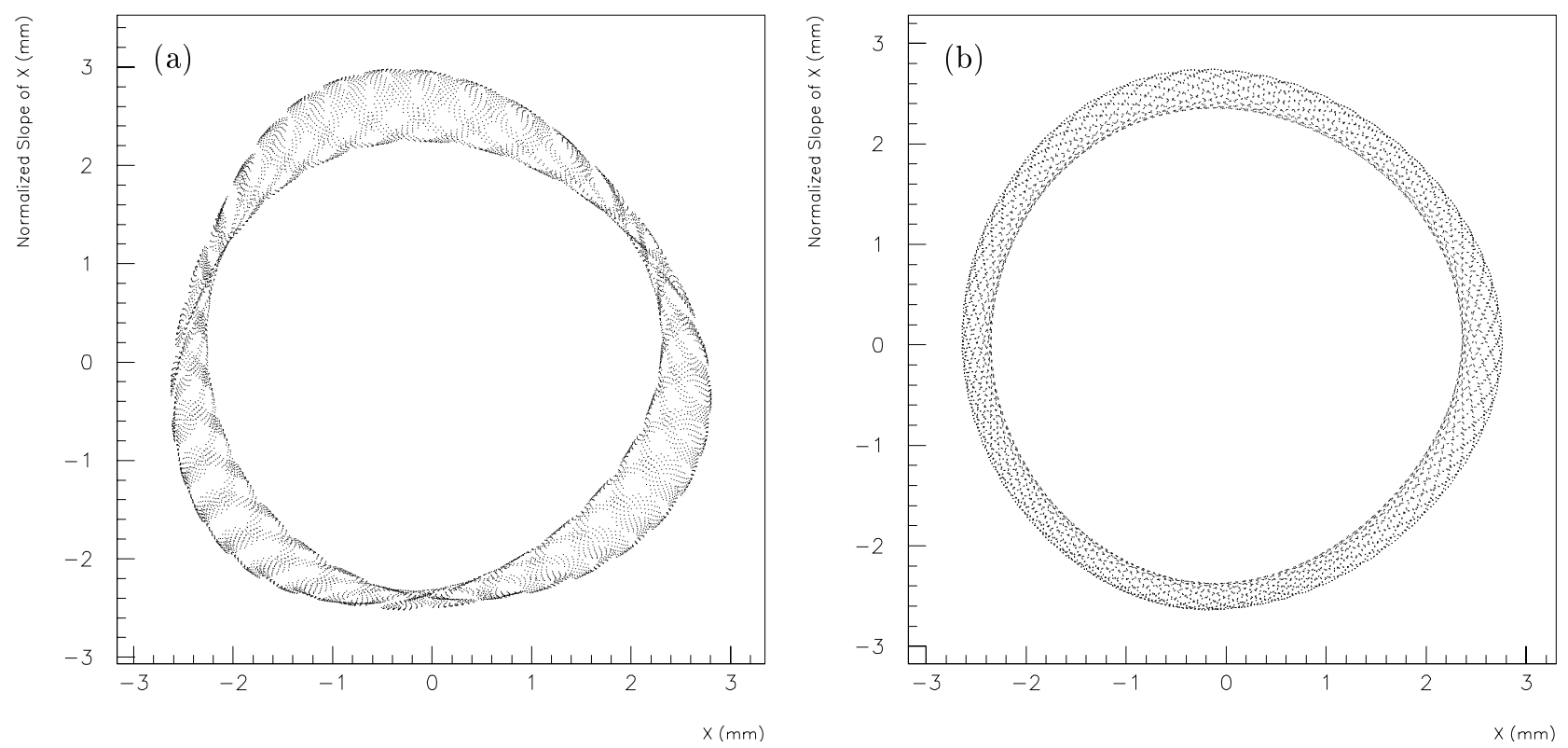

Figure 1: Reduction of phase space distortion due to the correction of the resonances. In part (a) the horizontal phase space of particle motion is shown in a LHC model with the $(3,0)$ and the $(1,2)$ resonances strongly excited. The phase space after correction is shown in part (b).

\section{Application in Tracking}

\subsection{Resonance Correction in the LHC}

A possible exploitation of the techniques consist in the correction of the resonance contributions generated by the nonlinear elements in an accelerator lattice. For this purpose tracking data of a realistic LHC model are analyzed.

Following well established strategies for the correction of the resonances [7] one has to to identify the location and the strength of a set of correctors families to compensate the third order resonances $(3,0)$ and $(1,2)$. A family of sextupolar spool pieces, normally used to correct the average $b_{3}$ component along the lattice was split into several families to compensate the cosine and sine term of the two resonances. Using tracking data at each location of the correctors the best places for correctors could be identified, i.e. locations where the spectral resonance lines have their maxima. Two resonances were corrected simultaneously each with two correction families while keeping the $b_{3}$ corrected on average. In this way the amplitude of the lines could be reduced by more that $50 \%$. The resulting reduction of the phase space distortions is clearly visible in Fig. 1. The double resonance correction leads to an improvement of the dynamic aperture of almost $10 \%$ (see Tab. 2). 
Table 2: Dynamic aperture improvement with correction of the $(3,0)$ and $(1,2)$ resonances in LHC. The stability border is given in units of the transverse rms beam size.

\begin{tabular}{lcc}
\hline \hline stability border & uncorrected & corrected \\
\hline \hline regular motion & 15.5 & 16.9 \\
strong chaos & 16.0 & 17.1 \\
Loss before 1000 turns & 16.9 & 18.0 \\
\hline \hline
\end{tabular}

\section{Experiments at RHIC}

An analysis of turn-by-turn data should allow to measure all linear and nonlinear accelerator properties relevant to single particle dynamics. Such properties are

- The base advance between pickups

- $\beta$-beating

- Linear coupling

- Chromaticity

- Detuning versus amplitude

- Driving terms of resonances

Ultimately, the full nonlinear model of the accelerator can be constructed. In 2000 we aimed at analyzing the effect of a local interaction region sextupole corrector on the detuning with amplitude and the resonance driving term of the third order resonance $(3,0)$.

Data were taken with all BPMs around the ring for 1024 turns. The horizontal beam oscillation amplitude was varied by injecting beam under different angles. For this the last horizontal corrector in the transfer line was set at different values.

The base tunes of the machine were $(28.105,29.112)$. The tune was optimized for polarized proton operation and could not be changed for the measurements due to the limited study time. To measure the effect of sextupolar disturbances in the lattice on the $(3,0)$ resonance, the base tune should be ideally close to this resonance line.

In Fig. 2 the amplitude dependent detuning is shown for four different values of the sextupole corrector, ranging from $+4 \mathrm{~A}$ to $-8 \mathrm{~A}$. The amplitude dependent tune shift clearly changes with the sextupole strength. The measurements with negative sextupole current setting also show that the amplitude dependent tune shift is linear with the square of the oscillation amplitude, as expected from theory [7].

Fig. 3 shows the ratio of the $(3,0)$ resonance amplitude to the $(1,0)$ resonance amplitude (the base tune) in the spectrum. Unlike in Fig. 2 the dependence of the curves on the strength of the sextupole corrector is less clear. The line amplitude ratio seems to follow a linear dependence on the amplitude, as expected from Tab. 1. Due to the weak dependence of the sextupole strength on the line amplitude ratio, resonance driving terms cannot be determined accurately. 


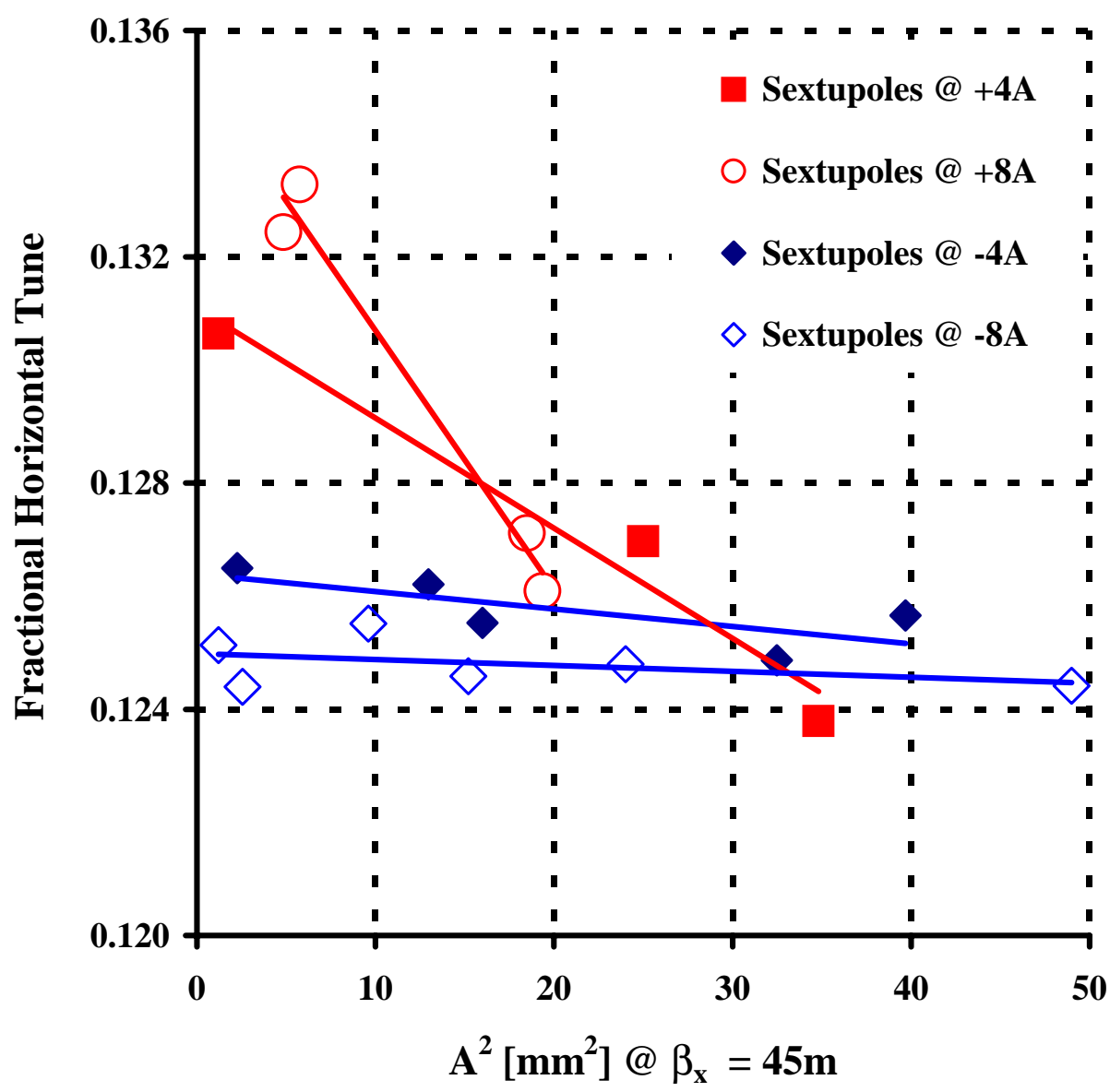

Figure 2: Detuning as function of amplitude.

\section{CONCLUSIONS}

A clear dependence of a single interaction region sextupole corrector on the amplitude dependent tune shift has been observed. An attempt to determine resonance driving terms from amplitude ratios of resonance lines in the spectra of turn-by-turn data was, however, unsuccessful. We think that the base tune was too far from the resonance under investigation. The signal in the spectrum was therefore too weak. Should we be able to measure resonance driving terms from a single corrector with different base tunes, the method would have potential for a beam based adjustment of nonlinear IR correctors.

\section{References}

[1] J. Laskar, "Secular evolution of the solar system over 10 million years", Astron. Astrophys. 198, pp. 341-362 (1988). 


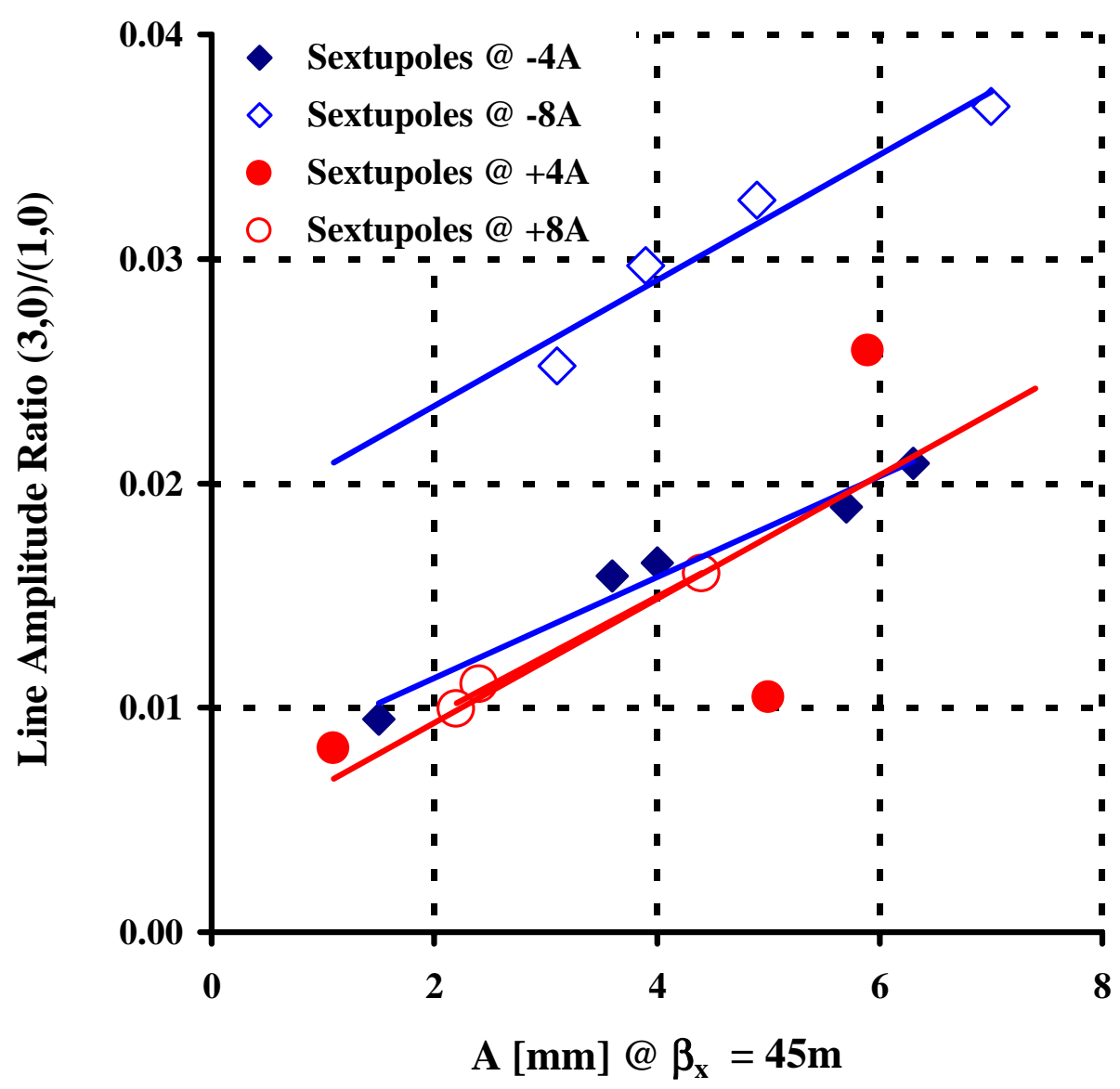

Figure 3: Line amplitude ratio of the resonances $(3,0)$ to $(1,0)$ as function of amplitude.

[2] R. Bartolini, A. Bazzani, M. Giovannozzi, W. Scandale, and E. Todesco, "Tune evaluation in simulations and experiments", Part. Acc. 56, pp. 167-199 (1996).

[3] J. Bengtsson, "Non-Linear transverse dynamics for storage rings with applications to the low-energy antiproton ring (LEAR) at CERN", CERN 88-05, (1988).

[4] R. Bartolini and F. Schmidt, "Normal Form via Tracking or Beam Data", LHC Project Note 132 (revised December 1999), Part. Accel. 59, pp. 93-106, (1998), http://wwwslap.cern.ch/frs/report/lines97.ps.gz.

[5] F. Schmidt, "Measurement of Resonance Driving Terms at the CERN SPS using turnby-turn data from beam position monitors", this conference.

[6] M. Berz, É. Forest and J. Irwin, "Normal form methods for complicated periodic systems: a complete solution using differential algebra and lie operators", Part. Acc. 24, pp. 91-107 (1989).

[7] G. Guignard, "A General Treatment of Resonance in accelerators", CERN 76-12, 1976. 